\title{
Defect sizing, separation, and substrate effects in ion-irradiated monolayer two-dimensional materials
}

\author{
Pierce Maguire, ${ }^{1,2}$ Daniel S. Fox, ${ }^{1,2}$ Yangbo Zhou ${ }^{1,2,3}$ Qianjin Wang, ${ }^{4}$ Maria O’Brien, ${ }^{2,5}$ Jakub Jadwiszczak, ${ }^{1,2}$ \\ Conor P. Cullen, ${ }^{2,5}$ John McManus, ${ }^{2,5}$ Samuel Bateman, ${ }^{1,2}$ Niall McEvoy, ${ }^{2,5}$ Georg S. Duesberg, ${ }^{2,5,6}$ and Hongzhou Zhang ${ }^{1,2, *}$ \\ ${ }^{1}$ School of Physics, Trinity College Dublin, Dublin 2, D02 PN40, Ireland \\ ${ }^{2}$ AMBER Centre, CRANN Institute, Trinity College Dublin, Dublin 2, D02 PN40, Ireland \\ ${ }^{3}$ School of Material Science and Engineering, Nanchang University, Youxun W Rd, Xinjian Qu, Nanchang Shi, \\ 330031 Jiangxi Sheng, People's Republic of China \\ ${ }^{4}$ National Laboratory of Solid State Microstructures, Nanjing University, Nanjing 210093, Jiangsu Province, People's Republic of China \\ ${ }^{5}$ School of Chemistry, Trinity College Dublin, Dublin 2, D02 PN40, Ireland \\ ${ }^{6}$ Institute of Physics, EIT 2, Faculty of Electrical Engineering and Information Technology, Universität der Bundeswehr München, \\ Werner-Heisenberg-Weg 39, 85577 Neubiberg, Germany
}

(Received 27 July 2017; revised manuscript received 25 September 2018; published 18 October 2018)

\begin{abstract}
Precise and scalable defect engineering of two-dimensional (2D) nanomaterials is acutely sought after in contemporary materials science. Here, we present defect engineering in monolayer graphene and molybdenum disulfide $\left(\mathrm{MoS}_{2}\right)$ by irradiation with noble gas ions at $30 \mathrm{keV}$. Two ion species of different masses were used in a gas field ion source microscope: helium $\left(\mathrm{He}^{+}\right)$and neon $\left(\mathrm{Ne}^{+}\right)$. A detailed Raman spectroscopy study was performed and a defect activation model applied with marked differences between the ion systems at a given dose. We propose that disparities between the ion systems are explained by different defect yields and defect sizes. Expanding on existing models, we suggest that the average defect size is smaller for supported than freestanding graphene and that the rate of defect production is larger. We infer that low-energy secondary atoms from the substrate play a significant role in defect production, creating smaller defects relative to those created by the primary ion beam. Furthermore, a similar model was also applied to supported $\mathrm{MoS}_{2}$, another promising member of the 2D material family. Defect yields for both ions were obtained for $\mathrm{MoS}_{2}$, demonstrating their different interaction with the material and facilitating comparison with other irradiation conditions in the literature.
\end{abstract}

DOI: 10.1103/PhysRevB.98.134109

\section{INTRODUCTION}

In recent years, the extraordinary properties and tunability of two-dimensional (2D) materials have been repeatedly demonstrated, heralding a new era of materials science [1,2]. Their physical properties (electrical, thermal, etc.) are highly distinguished from their bulk counterparts due to the evolution of band structure with decreasing layer number [3-6]. The ideas and methodologies developed from the investigation of graphene have been extended to many other 2D materials, including transition metal dichalcogenides (TMDs) such as molybdenum disulfide $[7,8]$.

With the demands of modern semiconductor technology, precise nanoscale control of $2 \mathrm{D}$ material properties is fervently pursued. The alteration of nanoscale geometry and the introduction of structural defects or strain to these materials can be used to modulate their electronic and optical properties [9-16]. Ion irradiation has an illustrious record over many decades in the scalable and precise defect engineering of materials $[17,18]$. Modern ion-irradiation techniques with subnanometer probe sizes have demonstrated sub-10-nm precision in fabricating nanostructures from various materials [19-26].

*Corresponding author: Hongzhou.Zhang@tcd.ie
Ultimate modification precision is the convolution of the impact probe and the interaction volume [27]. Given the superlative confinement of 2D materials in the out-of-plane direction, and the confinement of modern ion beams in the inplane direction, it is possible to restrict the ion-specimen interaction to an exceptionally small volume. Such methods have been used to control doping by implantation and to introduce precise quantities of defects $[14,24,28-31] . \mathrm{He}^{+}$irradiation of graphene encapsulated in hexagonal boron nitride has been used to introduce $n$-type doping [32] while dose-dependent irradiation of TMDs has introduced pseudometallic phases in the monolayer limit [24,33].

This paper reports an experimental comparison of two ion bombardment species, $\mathrm{He}^{+}$and $\mathrm{Ne}^{+}$, at high energy. It is also one of very few papers to carry out an explicit comparison between supported and freestanding 2D materials. The introduction of defects by ion irradiation is shown to be highly precise, and Raman spectroscopy proves to be a highly versatile and sensitive characterization method for these defective monolayer materials.

\section{A. Defect engineering by ion irradiation in 2D materials}

Defects in 2D materials such as a graphene and $\mathrm{MoS}_{2}$ have been introduced by a range of energetic species. For the 
application of such ion-irradiation methods to defect engineering, the defect yield per ion and the types of defect introduced must be known. The mass and energy of the primary ion species both play a substantial role in determining the average defect yield and the nature of the dominant defect type. With increased mass of energetic noble gas ions incident on graphene, both the defect yield and size are expected to increase [34-36]. For high-energy ions, the defect yield decreases with increasing energy while the defect size increases [34].

However, defects induced in supported 2D materials are created not just by the primary ion beam. A substrate is expected to lower the damage probability per ion for low-energy particles [37]. However, the converse is typically (though not always) found to be true for high-energy ions as the substrate facilitates backscattering of the primary beam and thus provides a source of energized secondary particles [38-43]. Zhao et al. found that irradiation damage was enhanced in supported graphene (compared to freestanding) for energies above $5 \mathrm{keV}$ for $\mathrm{Ar}^{+}(M=18)$ and $3 \mathrm{keV}$ for $\mathrm{Si}^{+}(M=14)$ [38]. Excited substrate particles have a lower energy than the primary ions which suggests that they would create defects of smaller size.

Lucchese et al. used low-energy $(90-\mathrm{eV}) \mathrm{Ar}^{+}$ions to alter the average distance between defects $L_{D}$ in graphene [13]. In these studies, $L_{D}$ was calculated based on the expected density of defects $\sigma$, which is estimated from the irradiation dose $S$ in ions per unit area. The approximation that $\sigma \simeq S$ was used which assumes a random distribution of ions, yielding $[13,29,30]$

$$
L_{D}=\frac{1}{\sqrt{\sigma}} .
$$

This is a valid assumption for ions with a suitable cross section for creating a single carbon vacancy defect. The 90-eV $\mathrm{Ar}^{+}$ ions applied to graphene by Lucchese et al. are one such example due to their relatively large mass and low energy. The vast majority of those ions do indeed interact with surface carbon atoms but due to the low energy can only remove one carbon atom each.

\section{B. Local activation models for Raman spectra of defective 2D materials}

The Raman spectra of graphene have been extensively studied $[13,14,30,44-50]$ with characteristic peaks labeled $2 D$ at $\sim 2640 \mathrm{~cm}^{-1}$ and $G$ at $\sim 1583 \mathrm{~cm}^{-1}$. In the Raman spectra of defective graphene, the disruption to normal selection rules also allows the detection of two additional peaks: $D$ at $\sim 1322 \mathrm{~cm}^{-1}$, and $D^{\prime}$ at $\sim 1600 \mathrm{~cm}^{-1}$ [44,51]. Furthermore, increasing structural disorder also causes the $G$ peak to consistently broaden. $L_{D}$ in graphene has been related to the ratio of the intensity of the $D$ peak $\left(I_{D}\right)$ to the intensity of the $G$ peak $\left(I_{G}\right)$ and a version of that relationship is given by Lucchese et al: :

$$
\begin{aligned}
\frac{I_{D}}{I_{G}}= & C_{A} \frac{\left(L_{S}^{2}+2 r_{S} L_{S}\right)}{\left(L_{S}^{2}+2 r_{S} L_{S}-r_{S}^{2}\right)}\left[e^{-\pi r_{S}^{2} / L_{D}^{2}}-e^{-\pi\left(L_{S}^{2}+2 r_{S} L_{S}\right) / L_{D}^{2}}\right] \\
& +C_{S}\left[1-e^{-\pi r_{S}^{2} / L_{D}^{2}}\right],
\end{aligned}
$$

where $r_{S}$ and $r_{A}$ represent the radii of a structurally disordered region created by an ion and of an outer $D$ band-activated area, respectively. $L_{S}=r_{A}-r_{S}$ is the Raman relaxation length for the resonant Raman scattering. The intensity of the $D$ peak is proportional to the total area of crystalline graphene that is activated by local defects. Thus, as $L_{D}$ becomes low (i.e., the defect density increases and the material becomes less crystalline) the $D$ band intensity falls due to the overlapping of the disordered areas and the decrease of the total $D$-activated area.

The dispersive effect of the excitation energy $E_{l}$ on the ratio of excitation of the $D$ and $G$ bands is included in the $C_{A}$ parameter, being a measure of the maximum possible value of the $I_{D} / I_{G}$ ratio [52]. Where $E_{l}$ is stated in $\mathrm{eV}, C_{A}$ has been given experimentally by [14]

$$
C_{A}=(160 \pm 48) \times E_{l}^{-4} .
$$

The $C_{S}$ parameter is the value of the $I_{D} / I_{G}$ ratio in the highly disordered limit and it is important in the large defect density regime $L_{D} \leqslant r_{S}[13,14]$. Typically, three stages are discussed in the evolution of the relationship described by Eq. (2). The first stage begins with pristine graphene. As isolated defects initially appear in the crystalline lattice, a rising $D$ peak is observable, increasing $I_{D} / I_{G}$. The second stage features red-shifting and continued broadening of the $G$ peak and a now diminishing $D$ peak. It is reached when defects coalesce and carbon valence declines [13,14,30,48]. The third stage is marked by the transition of the specimen to amorphous carbon bearing limited resemblance to the original graphene.

Raman spectroscopy has been employed extensively in the characterization of $\mathrm{MoS}_{2}$ in various forms [53-60] including monolayer (of polytype $1 \mathrm{H}$, point group $D_{3 H}$ ) which has the labeled characteristic peaks: $E^{\prime}$ at $\sim 383 \mathrm{~cm}^{-1}$ and $A_{1}^{\prime}$ at $\sim 401 \mathrm{~cm}^{-1}$. In defective material, an additional peak, the $L A(M)$ at $\sim 227 \mathrm{~cm}^{-1}$ is found [8,61-63]. The $E^{\prime}$ peak comes from the intralayer, in-plane motion of Mo and $\mathrm{S}$ atoms with respect to each other and the $A_{1}^{\prime}$ peak comes from the intralayer, out-of-plane motion of $\mathrm{S}$ atoms [8,56,62,64,65].

The $L A(M)$ peak appears in nanoparticle/multilayer samples but exhibits no intensity in pristine monolayer $\mathrm{MoS}_{2}$ [56,58,59]. However, it intensifies quickly with increased defect density [29,59]. Since it is defect activated, Mignuzzi et al. draw an analogy between the $L A(M)$ peak in monolayer $\mathrm{MoS}_{2}$ and the $D$ peak in graphene as both represent a good measure of disorder when normalized [29]. The intensity of the $L A(M)$ peak, $I(L A)$, normalized to that of either the $E^{\prime}$ peak, $\frac{I(L A)}{I\left(E^{\prime}\right)}$, or the $A_{1}^{\prime}$ peak, $\frac{I(L A)}{I\left(A_{1}^{\prime}\right)}$, is related to the inverse square of $L_{D}$ by

$$
\frac{I(L A)}{I(X)}=\frac{C(X)}{L_{D}^{2}} .
$$

$X=E^{\prime}$ or $A_{1}^{\prime}$ depending on the peak studied. In the case where the Raman spectrum is acquired with a 532-nm laser, the following constants were reported from the fitting of experimental data: $C\left(E^{\prime}\right)=1.11 \pm 0.08 \mathrm{~nm}^{2}$ and $C\left(A_{1}^{\prime}\right)=$ $0.59 \pm 0.03 \mathrm{~nm}^{2}$. During defect engineering, the increase of these intensity ratios is attributed to two concomitant factors: (i) an increase in the absolute intensity of the defect-activated $L A(M)$ peak, and (ii) a decrease in the intensity of the $E^{\prime}$ and $A_{1}^{\prime}$ peaks attributed to the ablation of the specimen [29]. 


\section{EXPERIMENT}

\section{A. Preparation of monolayer 2D materials}

Chemical vapor deposition (CVD) was used to grow the graphene sample on copper foil [66]. The graphene was transferred onto a Si substrate using a polymer-assisted process as outlined previously [66,67]. The Si substrate had arrays of holes with a diameter of $\sim 2 \mu \mathrm{m}$ and depths of $>10 \mu \mathrm{m}$ as pictured in Fig. S2 of the Supplemental Material [68].

$\mathrm{MoS}_{2}$ was also prepared using a CVD technique [69]. $\mathrm{MoO}_{3}$ substrates were placed face up in a ceramic boat with a blank $\mathrm{SiO}_{2}$ substrate face down on top. This was situated in the center of the heating zone of a quartz tube furnace, and ramped to $750^{\circ} \mathrm{C}$ under $150 \mathrm{SCCM}$ of Ar flow. Sulfur (S) vapor was then produced by heating $\mathrm{S}$ powder to $\sim 120^{\circ} \mathrm{C}$ in an independently controlled upstream heating zone of the furnace, and carried downstream to the $\mathrm{MoO}_{3}$ for a duration of $20 \mathrm{~min}$. After this, the furnace was held at $750{ }^{\circ} \mathrm{C}$ for $20 \mathrm{~min}$, then cooled down to room temperature. Monolayer flakes of $\mathrm{MoS}_{2}$ with a typical triangular shape could then be identified on the $\mathrm{SiO}_{2}$ surface by optical contrast.

\section{B. Irradiation with $30-\mathrm{keV} \mathrm{He}^{+}$and $\mathrm{Ne}^{+}$}

The Zeiss ORION NanoFab microscope was used to irradiate arrays of $5 \times 5 \mu \mathrm{m}^{2}$ regions in graphene and $\mathrm{MoS}_{2}$ with $\mathrm{He}^{+}$and $\mathrm{Ne}^{+}$at an energy of $30 \mathrm{keV}$ and an angle of incidence of $0^{\circ}$. These regions received doses ranging from $1.5 \times 10^{11}$ to $1 \times 10^{16} \mathrm{Ne}^{+} \mathrm{cm}^{-2}$ or $1 \times 10^{13}$ to $1 \times 10^{17} \mathrm{He}^{+} \mathrm{cm}^{-2}$. The beam was defocused $(\sim 10 \mathrm{~s}$ of $\mathrm{nm})$ to ensure a uniform distribution of ions and the sample was irradiated at the desired dose. 1-pA beam current and 10-nm pixel spacing were used. The beam dwell time at each pixel and/or the number of repeats at each position were varied to achieve the desired dose. The chamber pressure was of the order of $3 \times 10^{-7}$ Torr.

\section{Raman spectroscopy}

Raman spectroscopy was carried out on graphene with a Horiba Jobin-Yvon system (633-nm laser) with a 1200 lines/mm diffraction grating and a $100 \times$ objective aperture $(\mathrm{NA}=0.66)$ (laser spot size was $\sim 0.7 \mu \mathrm{m}$ ). These spectra were comprised of 10 acquisitions, each of 1-s duration at a single point for each irradiated region.

Raman spectroscopy was carried out on $\mathrm{MoS}_{2}$ using a WITec Alpha 300R system (532-nm laser) with a 1800 lines $/ \mathrm{mm}$ diffraction grating and a $100 \times$ objective $(\mathrm{NA}=$ 0.95 ) (laser spot size was $\sim 0.3 \mu \mathrm{m}$ ). Raman maps were generated by taking four spectra per $\mu \mathrm{m}$ in both $x$ and $y$ directions over large areas [70]. The acquisition time was $0.113 \mathrm{~s}$. The spectra from a desired region were acquired by averaging.

For both materials, the laser power was $\sim 1 \mathrm{~mW}$ to minimize sample damage. Peaks in the Raman spectra were fitted with Lorentzian functions for graphene. For $\mathrm{MoS}_{2}$, Gaussian functions were used for the $E^{\prime}$ and $A_{1}^{\prime}$ peaks and Lorentzians for the region around the $L A(M)$ peak (demonstrated in Fig. S6). Error bars, where used and unless otherwise stated, are the largest of either the instrumental $\left(1 \mathrm{~cm}^{-1}\right)$ or the fitting error as acquired from the FITYK software package, which uses a weighted sum of squared residuals to measure agreement between the fit and the data [71].

\section{RESULTS}

In Fig. 1, four sets of Raman spectra are presented, labeled as follows: (a) $\mathrm{He}^{+}$irradiated freestanding graphene, (b) $\mathrm{He}^{+}$ irradiated supported graphene, (c) $\mathrm{Ne}^{+}$irradiated freestanding graphene, and (d) $\mathrm{Ne}^{+}$irradiated supported graphene. The spectra obtained from the nonirradiated regions of both supported and freestanding samples are shown in black and agree with the literature for monolayer graphene [44,47].

In each subplot, multiple spectra are shown with dose increasing in ascent from the bottom. The first spectrum (black) represents the nonirradiated graphene. In the second spectrum (red), the defect-activated $D$ peak (at $\sim 1322 \mathrm{~cm}^{-1}$ ) can be observed to have increased in intensity relative to the $G$ band intensity. The third spectrum (green) shows a very intense $D$ peak. The $D^{\prime}$ peak is visible in some of the intermediate doses but was not studied in detail as it occurs only in a narrow dose range and has not yet been correlated to the defect activation model. Finally, in the fourth spectrum (blue) the material is amorphous carbon with little to no remaining crystallinity.

The width of the $G$ peak $\left(\Gamma_{G}\right)$ increases as a function of ion dose as shown in Fig. 2(a) for both $\mathrm{He}^{+}$and $\mathrm{Ne}^{+}$. For each type of sample and choice of ion this represents an unequivocal increase in structural disorder. While the trends for both ions are similar, the incidence of the $\mathrm{Ne}^{+}$species causes changes in the $G$ peak to occur at ion doses which are lower by between one and two orders of magnitude than those of $\mathrm{He}^{+}$. It should be noted that the difference between the effects of ion irradiation on $\Gamma_{G}$ for supported and freestanding graphene is very small here and difficult to separate from the direct effect of the substrate/suspension on the $G$ peak $[47,72]$. The fit to the data is of the form

$$
\Gamma_{G}=\Gamma_{G 0}+b S^{c}
$$

where $\Gamma_{G 0}$ is the width of the $G$ peak in pristine graphene, $b$ is a fitting parameter related to the defect yield, $S$ is the ion dose, and $c$ is a fitting parameter related to the effect of defect density on the $G$ peak. $c$ is found to be less than 1 in all cases, suggesting that the relationship between dose and $\Gamma_{G}$ is sublinear.

In Fig. 2(b), the evolution of the $I_{D} / I_{G}$ ratio against dose is displayed for both $\mathrm{He}^{+}$and $\mathrm{Ne}^{+}$and for both freestanding and supported graphene. Progression through the three previously discussed stages is observed. $I_{D} / I_{G}$ of the supported graphene is noted to rise faster and reach a much higher maximum than the freestanding material. The maximum $I_{D} / I_{G}$ ratio is also observed to be lower for freestanding $\mathrm{Ne}^{+}$irradiated graphene than $\mathrm{He}^{+}$irradiated graphene but larger for supported $\mathrm{Ne}^{+}$irradiated graphene than freestanding $\mathrm{Ne}^{+}$irradiated graphene. This is discussed in relation to defect sizes later.

The effects of $\mathrm{Ne}^{+}$irradiation are again shown to be dominant in a much lower dose range due to the enhanced ability of the species to create defects at and near the surface. By the binary collision approximation, it is expected that the increased mass of the ion will substantially increase its cross section for interaction with carbon atoms in the surface graphene layer and silicon atoms near the surface, therefore producing more recoil atoms and thus creating vastly more defects per ion than $\mathrm{He}^{+}$(see Supplemental Material [73]) [17]. 




(a) $\mathrm{He}^{+}$Irradiated Freestanding Graphene

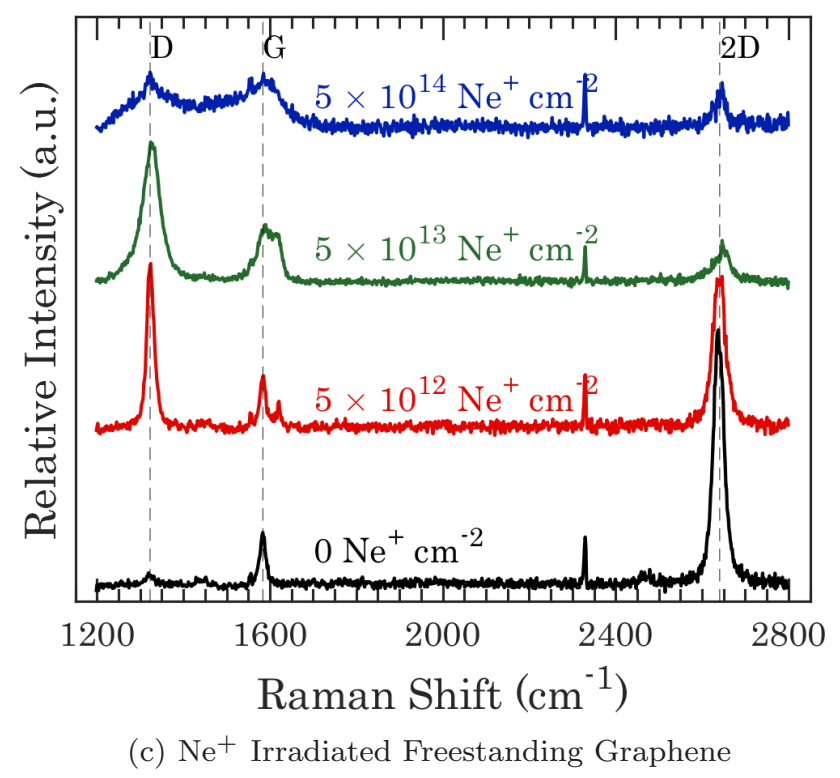

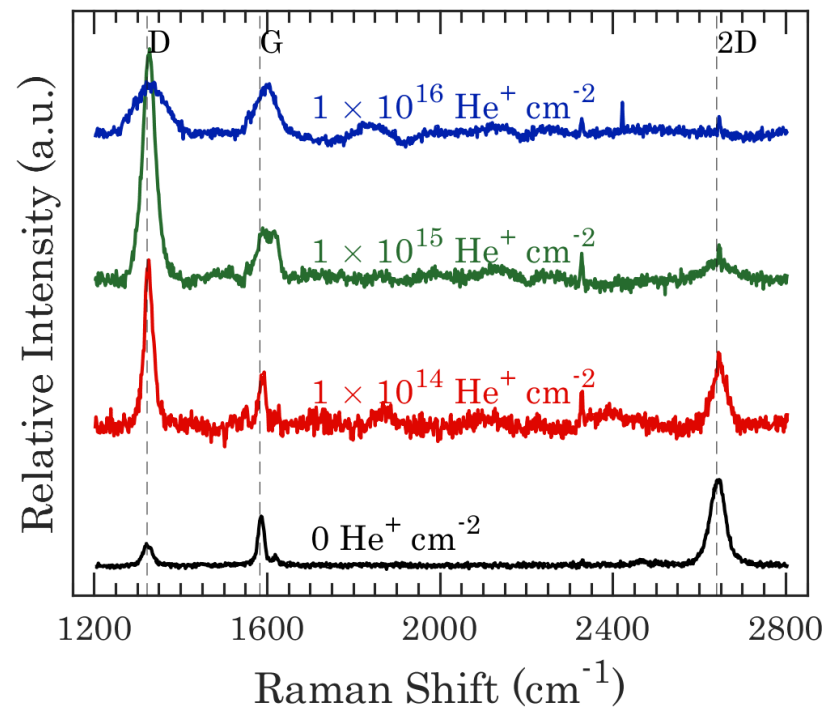

(b) $\mathrm{He}^{+}$Irradiated Supported Graphene

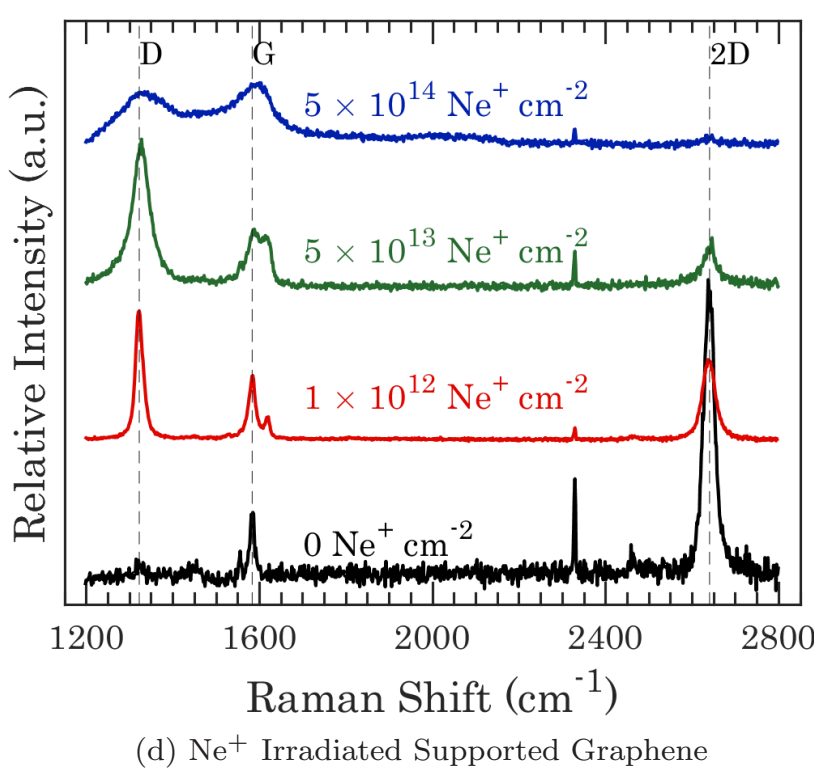

FIG. 1. Representative selection of graphene Raman spectra excited by a $633-\mathrm{nm}$ laser and irradiated with ions at $30 \mathrm{keV}$ with a $0^{\circ}$ angle of incidence. (a) Freestanding graphene irradiated with $\mathrm{He}^{+}$, (b) supported graphene irradiated with $\mathrm{He}^{+}$, (c) freestanding graphene irradiated with $\mathrm{Ne}^{+}$, (d) supported graphene irradiated with $\mathrm{Ne}^{+}$. The evolution of the spectra with increased ion dose is shown ascending from the bottom in black to the top in blue. The spectra are normalized to the maximum of the $G$ peak.

The data presented in Fig. 2(b) are also shown in Figs. 3(a) and 3(b) for $\mathrm{He}^{+}$and $\mathrm{Ne}^{+}$against $L_{D}$ as calculated from the included fit of Eq. (2). Further details and interpretation are provided in the discussion.

In Figs. 4(a) and 4(b), the characteristic $E^{\prime}$ and $A_{1}^{\prime}$ peaks of monolayer $\mathrm{MoS}_{2}$ are marked by dark gray dashed lines. In the pristine spectra (in black) they are in good agreement with the literature and the small separation of these two peaks $\left(\sim 18 \mathrm{~cm}^{-1}\right)$ is indicative of monolayer $\mathrm{MoS}_{2}$. With increasing ion dose, quenching and broadening of these two characteristic peaks are observed, reflecting the growing disorder which the ion beams create in the material. The emergence of the $L A(M)$ peak at $\sim 227 \mathrm{~cm}^{-1}$ (dashed gray line), particularly at high doses of $\mathrm{He}^{+}$and $\mathrm{Ne}^{+}$, is evident in Fig. 4 .
The evolutions of width and position of the $E^{\prime}$ and $A_{1}^{\prime}$ peaks are shown in Figs. 5(a) and 5(b), respectively. Both peaks are observed to broaden with increasing disorder and the peak positions shift as expected from previous reports [74]. Broadening begins at a substantially lower dose for $\mathrm{Ne}^{+}$than for $\mathrm{He}^{+}$as expected. The $E^{\prime}$ peak red-shifts and this downward shift in energy is attributed to the introduction of defects causing lattice distortion, similarly to tensile strain $[8,60,62,75-78]$. The $A_{1}^{\prime}$ peak also blue-shifts for some of the higher doses used, as previously reported [29].

Figure 6 (a) shows the evolution of the intensity ratios extracted from the spectra as a function of dose. With increasing disorder introduced by both ion beams, a sharp increase in the intensity of the $L A(M)$ peak normalized to both the $E^{\prime}$ and 


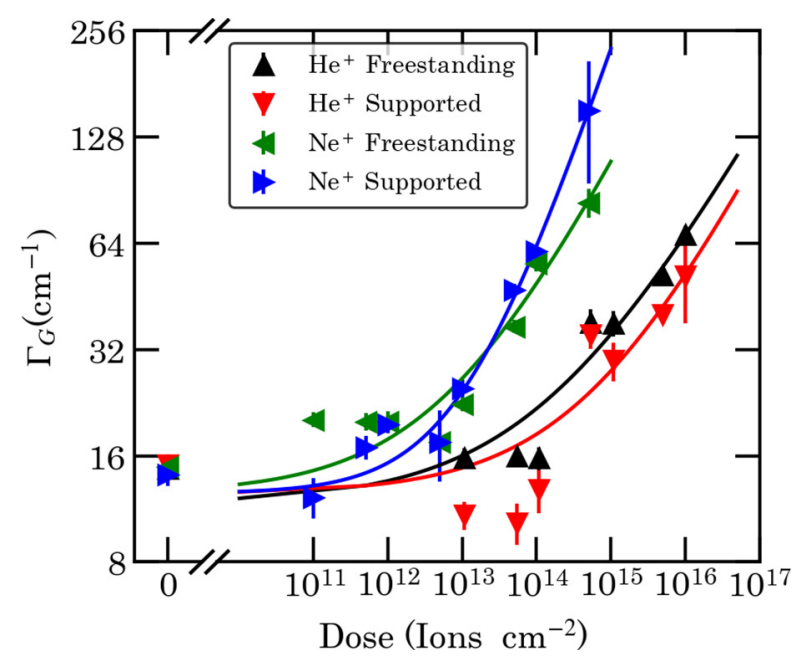

(a) Evolution of Width of the $G$ Peak with Ion Dose

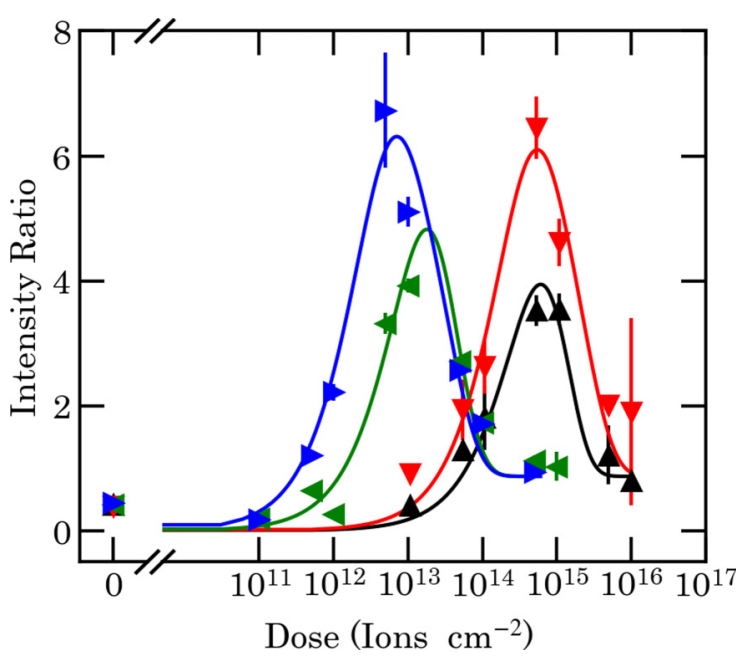

(b) Evolution of $\mathrm{I}_{D} / \mathrm{I}_{G}$ Ratio with Ion Dose

FIG. 2. (a) Evolution of full width at half-maximum of Lorentzian fits to the $G$ peak, $\Gamma_{G}$, as a function of dose for $\mathrm{He}^{+}$and $\mathrm{Ne}^{+}$for both freestanding and supported graphene. (b) The evolution of the $I_{D} / I_{G}$ ratio of graphene with irradiation dose. A version of Eq. (2) modified for dose has been fitted to each of the four data sets. The legend in (a) applies to both graphs.

$A_{1}^{\prime}$ peaks is observed. Once again, the increased defect yield of $\mathrm{Ne}^{+}$compared to $\mathrm{He}^{+}$is highlighted. Mignuzzi et al. used Eq. (4) to relate these intensity ratios directly to the average interdefect distance [29]. We have adjusted this approach slightly to account for the initial level of defects which is explained in the discussion.

\section{DISCUSSION}

We propose that the different effects of our ion-irradiation experiments on the $I_{D} / I_{G}$ ratio in graphene can be explained by considering the defect yield and defect size. The defect yield of the $\mathrm{Ne}^{+}$species at the incident surface is expected to

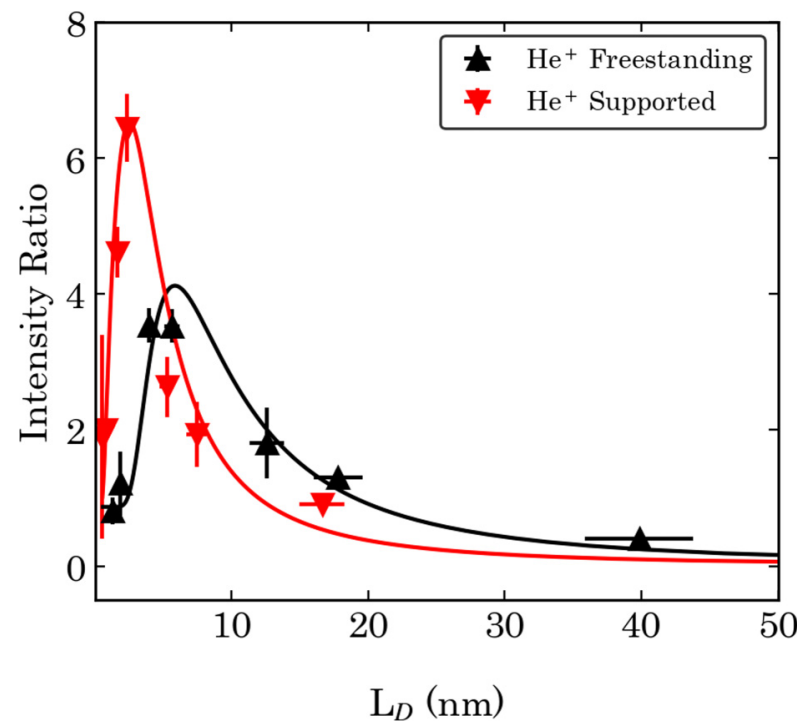

(a) Freestanding and Supported Graphene Irradiated with $\mathrm{He}^{+}$ be larger compared to $\mathrm{He}^{+}$due to its larger mass (and resulting higher cross section for interaction) [79].

\section{A. Defect yield}

For lighter and/or higher-energy ions such as those used in this work, the assumption made by Lucchese et al. that one ion produces one defect is clearly no longer valid [13]. In those cases, the average distance between defects [Eq. (1)] becomes

$$
L_{D}=\frac{1}{\sqrt{\alpha S}}
$$



(b) Freestanding and Supported Graphene Irradiated with $\mathrm{Ne}^{+}$

FIG. 3. The evolution of the $I_{D} / I_{G}$ ratio of freestanding and supported graphene with $L_{D}$ for (a) $\mathrm{He}^{+}$and (b) $\mathrm{Ne}^{+}$. The points represent experimental data and the lines are from a fit to Eq. (2) where $C_{A}$ is set at 7.6 [13]. 

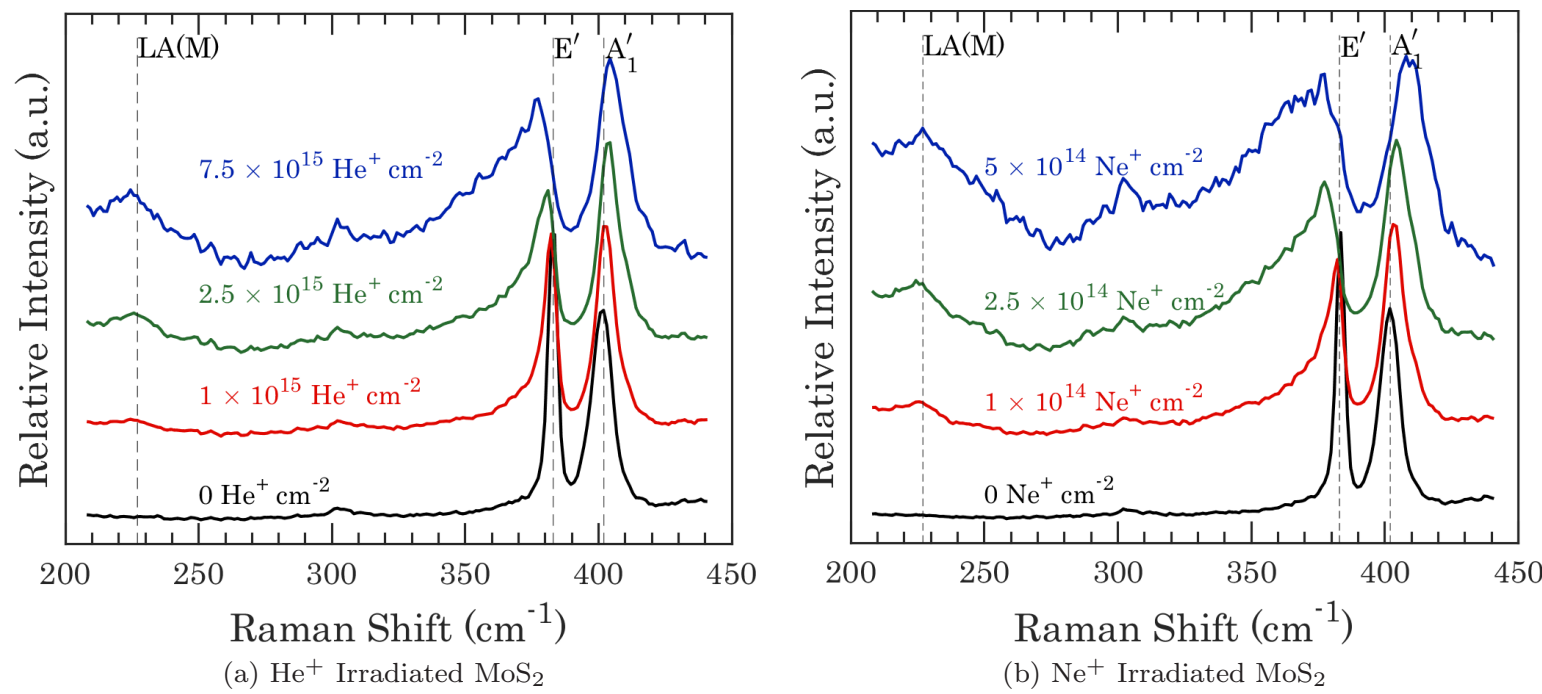

FIG. 4. A representative selection of Raman spectra of $\mathrm{MoS}_{2}$ with increased ion doses ascending from pristine at the bottom (in black) to the highest dose at the top (in blue). A 532-nm laser was used as the excitation source. Material irradiated with $\mathrm{He}^{+}$is shown in (a) and material irradiated with $\mathrm{Ne}^{+}$is shown in (b). Both ion species had an incident energy of $30 \mathrm{keV}$. The plots show the $L A(M)$ mode at $\sim 227 \mathrm{~cm}^{-1}$ as well as the $E^{\prime}$ and $A_{1}^{\prime}$ peaks. The spectra were individually normalized to the $A_{1}^{\prime}$ peak.

where $\alpha$ is the defect yield per ion and is distinguished from the sputtering yield $\gamma$ because not all defects causing local activation of Raman modes need necessarily be a vacancy.

Using this, a form of Eq. (2) was fitted to the four data sets in Figs. 5(a) and 5(b), showing the close agreement of the experimental data and the fitted equation. Initial values were provided from previous studies and from simulations. Since $C_{A}$ is only known imprecisely, the fit was performed for a range of values and the results are shown in Fig. S3 [80]. Results presented here were calculated using the lower bound value from Cançado et al. since this was found to lead to good agreement with literature [14]. The fitting process is described in more detail in the Supplemental Material [81].

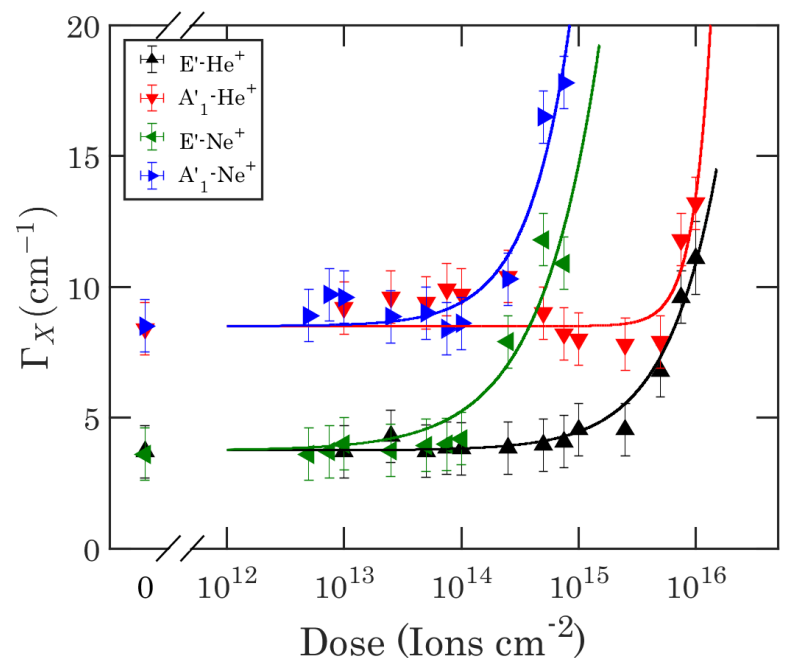

(a) FWHM of $E^{\prime}$ and $A_{1}^{\prime}$ peaks
Table I shows a variety of defect and sputtering yields from different methods for comparative purposes. The first are the SRIM (stopping and range of ions in matter) calculated values for sputtering yield $\left(\gamma_{C \text { (SRIM) }}\right)$, the second are the defect yields found by fitting to our experimental data $\left(\alpha_{C(\mathrm{fit})}\right)[38,82-$ 86]. The uncertainty quoted from the experimental data is the one standard deviation fitting error (discussed in detail in the Supplemental Material [87]). Two further values are included which are applicable to freestanding graphene only. $\gamma_{C(M D)}$ is the sputtering yield and $\alpha_{C(M D)}$ is the defect per ion value calculated using the molecular dynamics-derived online simulation of Lehtinen et al. [34,35].

The first point to note is that the $\mathrm{Ne}^{+}$ion universally has much larger yields, attributed to its greater mass. The second

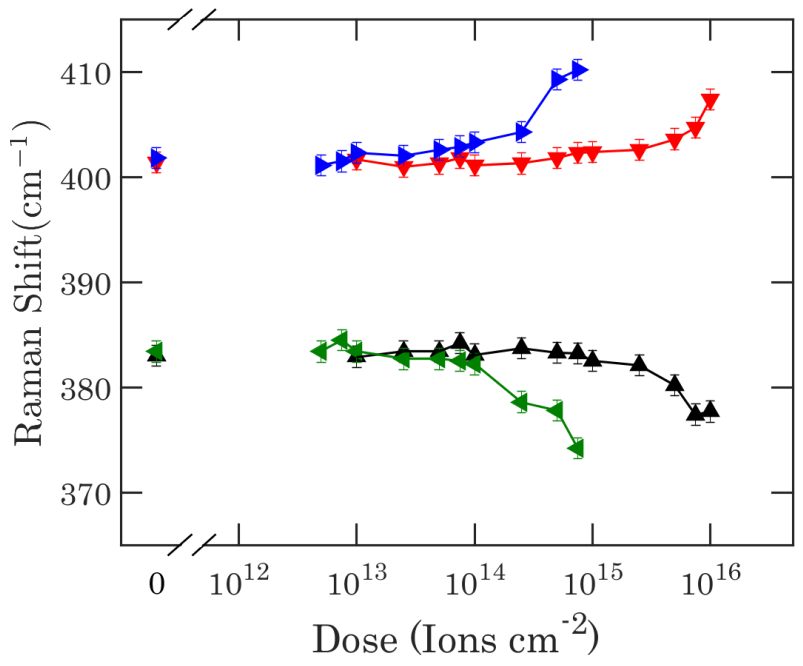

(b) Positions of $E^{\prime}$ and $A_{1}^{\prime}$ peaks

FIG. 5. (a) The evolution of the FWHM $\left(\Gamma_{X}\right)$ of $\mathrm{MoS}_{2}$ Raman modes as a function of ion dose. The data were fitted with a modified version of Eq. (5). (b) The evolution of peak position with ion dose. The legend in (a) applies to both graphs. 


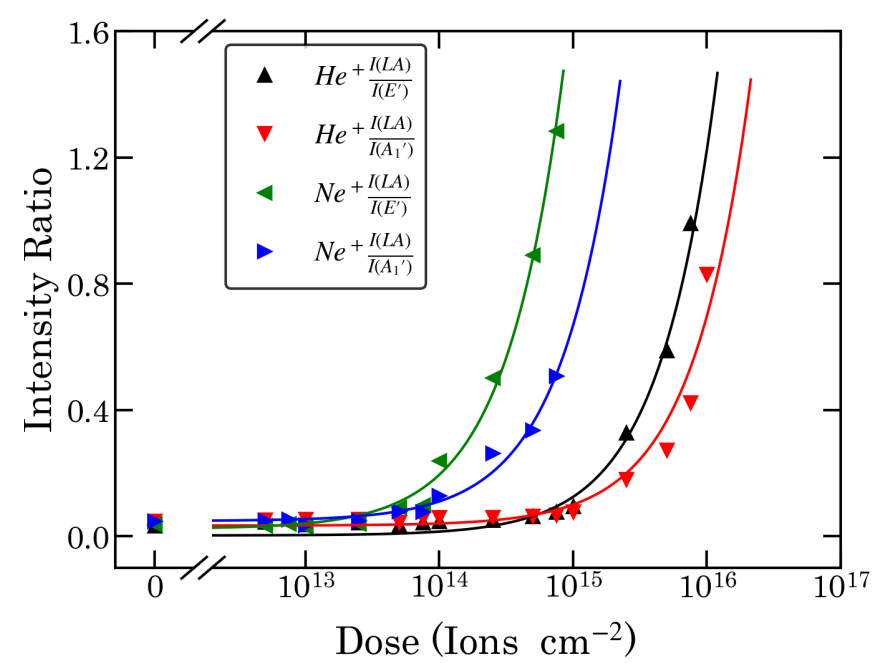

(a)

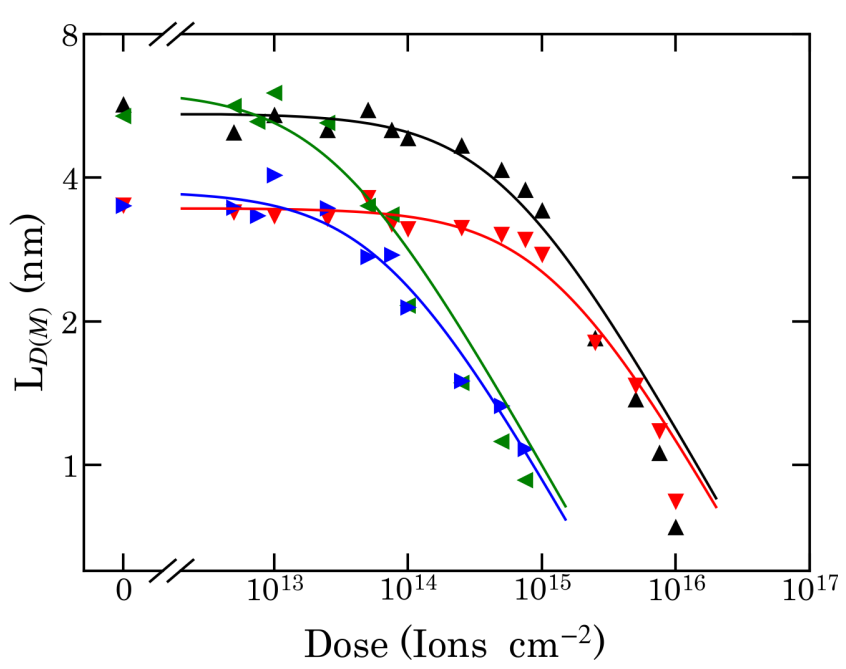

(b)

FIG. 6. (a) Shows the evolution with ion dose of the ratios of the intensity of the $L A(M)$ peak to the intensities of the $E^{\prime}$ and $A_{1}^{\prime}$ modes for both $\mathrm{He}^{+}$and $\mathrm{Ne}^{+}$irradiated $\mathrm{MoS}_{2}$. (b) Shows $L_{D(M)}$ as calculated from the intensity ratios in (a) and using the work of Mignuzzi et al. [29]. The legend in (a) applies to both graphs.

is that the sputtering and defect yields calculated for each ion are substantially larger in supported than freestanding graphene. This indicates that secondary particles from the substrate have a significant impact on the rate of defect introduction to the graphene layer.

In addition to the yields shown in the table, the sputtering yield of $\mathrm{He}^{+}$on freestanding graphene has also been measured experimentally before using a single pixel exposure to completely mill through a graphene layer. The sputtering yield value reported by Buchheim et al. is $\gamma_{C}=0.007$, which is in good agreement with atomistic simulations and our experimental result for defect yield [88]. It is also similar to the sputtering yield calculated using SRIM. A point to note is that for both $\mathrm{He}^{+}$and $\mathrm{Ne}^{+}$in freestanding graphene the experimentally derived $\alpha_{C \text { (fit) }}$ is smaller than the computationally derived $\alpha_{C(M D)}$, a discrepancy which is worthy of future study beyond this work.

\section{B. Defect sizes}

Aside from defect yield, we suggest that the other key factor to explain the $I_{D} / I_{G}$ ratio in graphene is the nature of the defects introduced. From Figs. 2(a) and 3 it is noted that the maximum $I_{D} / I_{G}$ value is considerably higher for supported graphene than for freestanding graphene. It is observed from Eq. (2) and displayed in Fig. S1 [89] that there is a close relationship between $r_{S}$ and the maximum of the $I_{D} / I_{G}$ ratio. From the previously mentioned fitting, we were also able to extract values for $r_{S}$.

Table II shows these values as well as the turning point coordinates. Depending on many factors, but principally the irradiation species, energy, and angle, we can generally expect $r_{S}$ values between approximately 0.8 and $2.5 \mathrm{~nm}[30,90]$. The values for $r_{S}$ are higher for freestanding than supported graphene. Lehtinen et al. found that the size of defects introduced by primary energetic particles typically increases with energy [34]. We suggest that while the higher-energy interaction of the direct beam is expected to cause large defects, it also excites substrate particles which have lower energies than the incident ion. These lower-energy atoms then produce smaller defect sites in the graphene at the surface.

The damage may partially anneal through thermalization and relaxation and the substrate may play a role in these processes. However, the established knowledge in ion-matter interactions can accurately predict the yield and energy of secondary atoms, leading to their contribution to the defect creation. This mechanism can explain the experimental data to a high degree of accuracy, and this indicates the thermalization and relaxation effects may be negligible for the ion doses used in our experiments.

It has been established that larger incident species usually produce larger defects $[30,34,35]$. However, in the freestanding case, we find that $r_{S}$ is unexpectedly not found to be larger for $\mathrm{Ne}^{+}$than $\mathrm{He}^{+}$, despite the very different ion mass. Although the uncertainty in our results is high here, this seemingly anomalous behavior may be related to a similarly unexpected experimental finding by Gawlik et al. and a

TABLE I. Sputtering yields $(\gamma)$ and defect per incident ion $\left(\alpha_{C}\right)$ values of carbon from graphene irradiated with $\mathrm{He}^{+}$and $\mathrm{Ne}^{+}$at $30 \mathrm{keV}$ and $0^{\circ}$ angle of incidence for the four different arrangements discussed in the main text. $\gamma_{C \text { (SRIM) }}$ is the sputtering yield calculated using SRIM, $\gamma_{C(M D)}$ is the sputtering yield calculated using the online simulation from Lehtinen et al. [34,35], $\alpha_{C \text { (fit) }}$ is the defect per ion value calculated from the fits shown in Fig. 3, and $\alpha_{C(M D)}$ is the defect per ion value calculated using the online simulation.

\begin{tabular}{lccccc}
\hline \hline Ion & Graphene & $\gamma_{C(\text { SRIM })}$ & $\gamma_{C(M D)}$ & $\alpha_{C(\mathrm{fit})}$ & $\alpha_{C(M D)}$ \\
\hline $\mathrm{He}^{+}$ & Freestanding & 0.010 & 0.006 & $0.006 \pm 0.003$ & 0.049 \\
$\mathrm{He}^{+}$ & Supported & 0.137 & & $0.033 \pm 0.015$ & \\
$\mathrm{Ne}^{+}$ & Freestanding & 0.236 & 0.156 & $0.41 \pm 0.33$ & 1.117 \\
$\mathrm{Ne}^{+}$ & Supported & 3.15 & & $1.45 \pm 0.55$ & \\
\hline \hline
\end{tabular}


TABLE II. Key parameters calculated from fitting Eq. (2) to graphene experimental data as in Fig. 3. These values are the average defect size $\left(r_{S}\right)$, the average distance between defects at which the maxiumum $\frac{I_{D}}{I_{G}}$ value occurred $\left[L_{D}\left(\frac{I_{D}}{I_{G}}\right)^{\max }\right]$, and the maximum $\frac{I_{D}}{I_{G}}$ value $\left[\left(\frac{I_{D}}{I_{G}}\right)^{\text {max }}\right] . C_{A}$ was fixed at 7.6 for this calculation, results from other values are presented in Fig. S3.

\begin{tabular}{lcccc}
\hline \hline Ion & Graphene & $r_{S}$ & $L_{D}\left(\frac{I_{D}}{I_{G}}\right)^{\max }$ & $\left(\frac{I_{D}}{I_{G}}\right)^{\max }$ \\
\hline $\mathrm{He}^{+}$ & Freestanding & $2.94 \pm 0.58 \mathrm{~nm}$ & $5.85 \mathrm{~nm}$ & 4.12 \\
$\mathrm{He}^{+}$ & Supported & $0.60 \pm 0.12 \mathrm{~nm}$ & $2.53 \mathrm{~nm}$ & 6.53 \\
$\mathrm{Ne}^{+}$ & Freestanding & $1.49 \pm 0.52 \mathrm{~nm}$ & $3.91 \mathrm{~nm}$ & 5.26 \\
$\mathrm{Ne}^{+}$ & Supported & $0.82 \pm 0.13 \mathrm{~nm}$ & $2.88 \mathrm{~nm}$ & 6.16 \\
\hline \hline
\end{tabular}

discrepancy found between computation and experiment by Yoon et al. [90,91]. This is worthy of future investigation.

A comparison of ion species, their mass, and the size of defects they introduce in supported graphene is provided in Table III. For supported graphene irradiated with $30-\mathrm{keV}$ $\mathrm{He}^{+}, r_{S}$ has been found to be $\sim 0.8-1 \mathrm{~nm}[22,48]$. The value obtained in this work for $\mathrm{He}^{+}$is slightly smaller than in those previous reports $[22,48]$. Since the mass of $\mathrm{Ne}^{+}$is between those of $\mathrm{He}^{+}$and $\mathrm{Ga}^{+}$, by a simple argument, it might be expected that the corresponding $r_{S}$ value would be similarly intermediate and this is indeed our finding. $\mathrm{Ga}^{+}$, also incident on supported graphene and also at $30 \mathrm{keV}$, has been reported to create defects of $r_{S}=1.6 \mathrm{~nm}$ [22]. Thus, the trend for supported graphene is clearly one of increasing defect size with increasing ion mass $m_{a}$.

Using the binary collision approximation $\left(m_{\mathrm{He}} / m_{\mathrm{Si}}=\right.$ 0.14 and $m_{\mathrm{Ne}} / m_{\mathrm{He}}=0.72$ ) we propose that the larger ion transfers energy more efficiently to substrate atoms near the surface. It is these particles, being more efficiently energized than their $\mathrm{He}^{+}$-induced counterparts, which create larger defects in the graphene layer. The variety of $r_{S}$ values obtained in this work suggests a variety of defect types with different weighting in the four experimental scenarios. Such defects may include single vacancies, double vacancies, complex defects, or amorphization [34,35]. This underscores the importance of choosing ion and substrate carefully for both nanofabrication and defect engineering.

\section{C. $\mathrm{MoS}_{2}$}

We highlight some caveats with regard to the approach of Mignuzzi et al. in addition to those present for graphene. Since $\mathrm{MoS}_{2}$ is nonmonoatomic, changes in stoichiometry may cause

TABLE III. Comparison of defect size $\left(r_{S}\right)$ in supported graphene caused by three ion species of different masses $\left(m_{a}\right)$ at $30 \mathrm{keV}$.

\begin{tabular}{lcc}
\hline \hline Ion & $r_{S}$ & $m_{a}$ \\
\hline $\mathrm{He}^{+}$ & $0.60 \pm 0.12 \mathrm{~nm}$ & 4 \\
$\mathrm{Ne}^{+}$ & $0.82 \pm 0.13 \mathrm{~nm}$ & 20.1 \\
$\mathrm{Ga}^{+}$ & $1.6 \mathrm{~nm}$ & $69.7[22]$ \\
\hline \hline
\end{tabular}

TABLE IV. (a) Initial defect density $\left(\sigma_{1}\right)$ and defect yields $\left(\alpha_{M}\right)$ calculated from fitting Eq. (7) to data as shown in Fig. 6(b).

\begin{tabular}{|c|c|c|c|c|c|}
\hline & \multicolumn{2}{|c|}{$\mathrm{He}^{+}$} & \multicolumn{2}{|c|}{$\mathrm{Ne}^{+}$} & \multirow[b]{2}{*}{ Units } \\
\hline & $E^{\prime}$ & $A_{1}^{\prime}$ & $E^{\prime}$ & $A_{1}^{\prime}$ & \\
\hline$\sigma_{1}$ & 3.3 & 8.4 & 2.7 & 7.1 & $1 \times 10^{12}$ defects $/ \mathrm{cm}^{2}$ \\
\hline$\alpha_{M}$ & 0.0067 & 0.0071 & 0.0976 & 0.1081 & Dimensionless \\
\hline
\end{tabular}

more complex defect-dependent behavior. Also, Mignuzzi et al. made an implicit assumption that each ion causes one defect which we do not consider to be safe for either the $25-\mathrm{keV} \mathrm{Mn}^{+}$used in their work or indeed the lighter $30-\mathrm{keV}$ ions used in this work [13]. Nonetheless, this form of the defect-activation model and the constants provided previously were applied to our data to calculate the average displacement between defects $L_{D(M)}$, for both peaks and for both $\mathrm{He}^{+}$and $\mathrm{Ne}^{+}$irradiation. In order to calculate the defect yield per ion $\alpha_{M}$, the initial defect level is accounted for in an adjusted version of Eq. (6):

$$
L_{D(M)}=\frac{1}{\sqrt{\alpha_{M} S+\sigma_{i}}},
$$

where $\sigma_{i}$ is the defect density in nonirradiated $\mathrm{MoS}_{2}$. These $L_{D(M)}$ values are presented as a function of ion dose in Fig. 6(b). Even the nonirradiated $\mathrm{MoS}_{2}$ has a somewhat low $L_{D(M)}$, not unusual for CVD-grown material. It is notable that in the high $L_{D(M)}$ range, where the ion dose is small, there is a discrepancy between the values given by the two peaks. Using $\frac{I\left(E^{\prime}\right)}{I(L A(M))}$ to calculate $L_{D(M)}$ yields a consistently higher value than using $\frac{I\left(A_{1}^{\prime}\right)}{I(L A(M))}$. However, the two values approach each other as $L_{D(M)}$ decreases which suggests that the nature of the initial defects may be different to those introduced by ion irradiation. Equation (7) allows for the extraction of the $\sigma_{i}$ and $\alpha_{M}$ values for both peaks and both ions as presented in Table IV. The values for $\sigma_{i}$ are in good agreement between the two ions, though as mentioned already do not agree between the two peaks. The values for $\alpha_{M}$ do agree well between peaks but, as expected, are very different for the two ions of such different masses.

\section{CONCLUSION}

In this study, we explained the effects of both $\mathrm{He}^{+}$and $\mathrm{Ne}^{+}$irradiation at $30 \mathrm{keV}$ on graphene and $\mathrm{MoS}_{2}$ using models of their Raman spectra. For both materials, doses above $5 \times 10^{15} \mathrm{~cm}^{-2}$ and $5 \times 10^{14} \mathrm{~cm}^{-2}$ for the respective ion species have resulted in severe changes in the spectra relative to the starting material. For both material systems, this severe breakdown occurs at a $\mathrm{Ne}^{+}$dose which is between one and two orders of magnitude less than that of $\mathrm{He}^{+}$. We believe this represents the first accurate experimental comparison of defect sizes produced in 2D materials by different noble gas ion probes. The dose dependence of irradiation species and relationships to interdefect distance have been established for graphene. A clear comparison to the literature is also 
reported for $\mathrm{MoS}_{2}$. In addition, we studied the effects of both primary ion species and secondary particles on the sizes of the defects produced in graphene. The role of substrate particles on defect production and size are also highlighted as a concern for nanofabrication methodologies due to the clear secondary atom effect. These results will allow a more informed and precise defect engineering of the investigated monolayer materials.

\section{ACKNOWLEDGMENTS}

We thank the staff at the Photonics and the Advanced Microscopy Laboratories (AML), CRANN, Trinity College Dublin. We are also grateful to the National Laboratory of Solid State Microstructures, Nanjing University. We acknowledge support from the following grants: Science Foundation Ireland (Grants No. 12/RC/2278, No. 11/PI/1105, No. 07/SK/I1220a, No. 15/IA/3131, and No. 08/CE/I1432).
[1] K. S. Novoselov, A. K. Geim, S. V. Morozov, D. Jiang, Y. Zhang, S. V. Dubonos, I. V. Grigorieva, and A. A. Firsov, Science 306, 666 (2004).

[2] K. S. Novoselov, V. I. Fal'ko, L. Colombo, P. R. Gellert, M. G. Schwab, and K. Kim, Nature (London) 490, 192 (2012).

[3] S. Z. Butler, S. M. Hollen, L. Cao, Y. Cui, J. A. Gupta, H. R. Gutiérrez, T. F. Heinz, S. S. Hong, J. Huang, A. F. Ismach, E. Johnston-Halperin, M. Kuno, V. V. Plashnitsa, R. D. Robinson, R. S. Ruoff, S. Salahuddin, J. Shan, L. Shi, M. G. Spencer, M. Terrones, W. Windl, and J. E. Goldberger, ACS Nano 7, 2898 (2013).

[4] R. Mas-Ballesté, C. Gómez-Navarro, J. Gómez-Herrero, and F. Zamora, Nanoscale 3, 20 (2011).

[5] C. Backes, R. J. Smith, N. McEvoy, N. C. Berner, D. McCloskey, H. C. Nerl, A. O’Neill, P. J. King, T. Higgins, D. Hanlon, N. Scheuschner, J. Maultzsch, L. Houben, G. S. Duesberg, J. F. Donegan, V. Nicolosi, and J. N. Coleman, Nat. Commun. 5, 4576 (2014).

[6] A. Splendiani, L. Sun, Y. Zhang, T. Li, J. Kim, C. Y. Chim, G. Galli, and F. Wang, Nano Lett. 10, 1271 (2010).

[7] K. F. Mak, C. Lee, J. Hone, J. Shan, and T. F. Heinz, Phys. Rev. Lett. 105, 136805 (2010).

[8] C. Lee, H. Yan, L. E. Brus, T. F. Heinz, J. Hone, and S. Ryu, ACS Nano 4, 2695 (2010).

[9] C. Ataca, H. Şahin, E. Aktürk, and S. Ciraci, J. Phys. Chem. C 115, 3934 (2011).

[10] M. Ghorbani-Asl, N. Zibouche, M. Wahiduzzaman, A. F. Oliveira, A. Kuc, and T. Heine, Sci. Rep. 3, 2961 (2013).

[11] Y. Zhou, Z. Liao, Y. Wang, G. S. Duesberg, J. Xu, Q. Fu, X. Wu, and D. Yu, J. Chem. Phys. 133, 234703 (2010).

[12] W. Zhou, X. Zou, S. Najmaei, Z. Liu, Y. Shi, J. Kong, J. Lou, P. M. Ajayan, B. I. Yakobson, and J. C. Idrobo, Nano Lett. 13, 2615 (2013).

[13] M. M. Lucchese, F. Stavale, E. H. Martins Ferreira, C. Vilani, M. V. O. Moutinho, R. B. Capaz, C. A. Achete, and A. Jorio, Carbon 48, 1592 (2010).

[14] L. G. Cançado, A. Jorio, E. H. Martins Ferreira, F. Stavale, C. A. Achete, R. B. Capaz, M. V. O. Moutinho, A. Lombardo, T. S. Kulmala, and A. C. Ferrari, Nano Lett. 11, 3190 (2011).

[15] H.-Q. Zhao, X. Mao, D. Zhou, S. Feng, X. Shi, Y. Ma, X. Wei, and Y. Mao, Nanoscale 8, 18995 (2016).

[16] H. P. Komsa, J. Kotakoski, S. Kurasch, O. Lehtinen, U. Kaiser, and A. V. Krasheninnikov, Phys. Rev. Lett. 109, 035503 (2012).

[17] M. Nastasi, J. W. Mayer, and J. K. Hirvonen, Ion-Solid Interactions: Fundamentals and Applications, 1st ed. (Cambridge University Press, Cambridge, 1996), pp. 62-87.
[18] S. Mathew, T. K. Chan, D. Zhan, K. Gopinadhan, A. R. Barman, M. B. H. Breese, S. Dhar, Z. X. Shen, T. Venkatesan, and J. T. L. Thong, Carbon 49, 1720 (2011).

[19] D. C. Bell, M. C. Lemme, L. A. Stern, J. R. Williams, and C. M. Marcus, Nanotechnol. 20, 455301 (2009).

[20] R. R. Nair, M. Sepioni, I. L. Tsai, O. Lehtinen, J. Keinonen, A. V. Krasheninnikov, T. Thomson, A. K. Geim, and I. V. Grigorieva, Nat. Phys. 8, 199 (2012).

[21] D. Fox, Y. B. Zhou, A. O’Neill, S. Kumar, J. Wang, J. N. Coleman, G. S. Duesberg, J. F. Donegan, and H. Zhang, Nanotechnol. 24, 335702 (2013).

[22] B. S. Archanjo, B. Fragneaud, L. G. Cançado, D. Winston, F. Miao, C. A. Achete, and G. Medeiros-Ribeiro, Appl. Phys. Lett. 104, 193114 (2014).

[23] N. Kalhor, S. A. Boden, and H. Mizuta, Microelectron. Eng. 114, 70 (2014).

[24] D. S. Fox, Y. Zhou, P. Maguire, A. O’Neill, C. O’Coileáin, R. Gatensby, A. M. Glushenkov, T. Tao, G. S. Duesberg, I. V. Shvets, M. Abid, M. Abid, H. C. Wu, Y. Chen, J. N. Coleman, J. F. Donegan, and H. Zhang, Nano Lett. 15, 5307 (2015).

[25] Y. Naitou, T. Iijima, and S. Ogawa, Appl. Phys. Lett. 106, 033103 (2015).

[26] S. Kim, O. Dyck, A. V. Ievlev, I. V. Vlassiouk, S. V. Kalinin, A. Belianinov, S. Jesse, and O. S. Ovchinnikova, Carbon 138, 277 (2018).

[27] R. Livengood, S. Tan, Y. Greenzweig, J. Notte, and S. McVey, J. Vacuum Sci. Technol. B 27, 3244 (2009).

[28] A. Nipane, D. Karmakar, N. Kaushik, S. Karande, and S. Lodha, ACS Nano 10, 2128 (2016).

[29] S. Mignuzzi, A. J. Pollard, N. Bonini, B. Brennan, I. S. Gilmore, M. A. Pimenta, D. Richards, and D. Roy, Phys. Rev. B 91, 195411 (2015).

[30] A. J. Pollard, B. Brennan, H. Stec, B. J. Tyler, M. P. Seah, I. S. Gilmore, and D. Roy, Appl. Phys. Lett. 105, 253107 (2014).

[31] M. G. Stanford, P. R. Pudasaini, A. Belianinov, N. Cross, J. H. Noh, M. Koehler, D. G. Mandrus, G. Duscher, A. J. Rondinone, I. N. Ivanov, T. Z. Ward, and P. D. Rack, Sci. Rep. 6, 27276 (2016).

[32] G. Nanda, S. Goswami, K. Watanabe, T. Taniguchi, and P. F. A. Alkemade, Nano Lett. 15, 4006 (2015).

[33] M. G. Stanford, P. R. Pudasaini, E. T. Gallmeier, N. Cross, L. Liang, A. Oyedele, G. Duscher, M. Mahjouri-Samani, K. Wang, K. Xiao, D. B. Geohegan, A. Belianinov, B. G. Sumpter, and P. D. Rack, Adv. Funct. Mater. 27, 1702829 (2017).

[34] O. Lehtinen, J. Kotakoski, A. V. Krasheninnikov, A. Tolvanen, K. Nordlund, and J. Keinonen, Phys. Rev. B 81, 153401 (2010). 
[35] O. Lehtinen, J. Kotakoski, A. V. Krasheninnikov, and J. Keinonen, Nanotechnol. 22, 175306 (2011).

[36] E. H. Ahlgren, J. Kotakoski, O. Lehtinen, and A. V. Krasheninnikov, Appl. Phys. Lett. 100, 233108 (2012).

[37] A. Zandiatashbar, G. H. Lee, S. J. An, S. Lee, N. Mathew, M. Terrones, T. Hayashi, C. R. Picu, J. Hone, and N. Koratkar, Nat. Commun. 5, 3186 (2014).

[38] S. Zhao, J. Xue, Y. Wang, and S. Yan, Nanotechnol. 23, 285703 (2012).

[39] A. V. Krasheninnikov, K. Nordlund, and J. Keinonen, Phys. Rev. B 65, 165423 (2002).

[40] W. Li, X. Wang, X. Zhang, S. Zhao, H. Duan, and J. Xue, Sci. Rep. 5, 9935 (2015).

[41] S. Kretschmer, M. Maslov, S. Ghaderzadeh, M. Ghorbani-Asl, G. Hlawacek, and A. V. Krasheninnikov, ACS Appl. Mater. Interfaces 10, 30827 (2018).

[42] C. L. Wu, H. T. Lin, H. A. Chen, S. Y. Lin, M. H. Shih, and C. W. Pao, Mater. Today Commun. 17, 60 (2018).

[43] J. J. Lopez, F. Greer, and J. R. Greer, J. Appl. Phys. 107, 104326 (2010).

[44] A. C. Ferrari, J. C. Meyer, V. Scardaci, C. Casiraghi, M. Lazzeri, F. Mauri, S. Piscanec, D. Jiang, K. S. Novoselov, S. Roth, and A. K. Geim, Phys. Rev. Lett. 97, 187401 (2006).

[45] A. C. Ferrari, Solid State Commun. 143, 47 (2007).

[46] Y. Wang, Z. Ni, T. Yu, Z. X. Shen, H. Wang, Y. Wu, W. Chen, A. Thye, and S. Wee, J. Phys. Chem. C 112, 10637 (2008).

[47] A. C. Ferrari and D. M. Basko, Nat. Nanotechnol. 8, 235 (2013).

[48] S. Hang, Z. Moktadir, and H. Mizuta, Carbon 72, 233 (2014).

[49] J. Ribeiro-Soares, M. E. Oliveros, C. Garin, M. V. David, L. G. Martins, C. A. Almeida, E. H. Martins-Ferreira, K. Takai, T. Enoki, R. Magalhães-Paniago, A. Malachias, A. Jorio, B. S. Archanjo, C. A. Achete, and L. G. Cançado, Carbon 95, 646 (2015).

[50] Y. Jia, X. Gong, P. Peng, Z. Wang, Z. Tian, L. Ren, Y. Fu, and H. Zhang, Nano-Micro Lett. 8, 336 (2016).

[51] L. M. Malard, M. A. Pimenta, G. Dresselhaus, and M. S. Dresselhaus, Phys. Rep. 473, 51 (2009).

[52] L. G. Cançado, A. Jorio, and M. A. Pimenta, Phys. Rev. B 76, 064304 (2007).

[53] T. J. Wieting and J. L. Verble, Phys. Rev. B 3, 4286 (1971).

[54] J. M. Chen and C. S. Wang, Solid State Commun. 14, 857 (1974).

[55] B. C. Windom, W. G. Sawyer, and D. W. Hahn, Tribol. Lett. 42, 301 (2011).

[56] T. Livneh and J. E. Spanier, 2D Mater. 2, 035003 (2015).

[57] X. Zhang, X. F. Qiao, W. Shi, J. B. Wu, D. S. Jiang, and P. H. Tan, Chem. Soc. Rev. 44, 2757 (2015).

[58] A. M. Stacy and D. T. Hodul, J. Phys. Chem. Solids 46, 405 (1985).

[59] G. L. Frey, R. Tenne, M. J. Matthews, M. S. Dresselhaus, and G. Dresselhaus, Phys. Rev. B 60, 2883 (1999).

[60] C. Rice, R. J. Young, R. Zan, U. Bangert, D. Wolverson, T. Georgiou, R. Jalil, and K. S. Novoselov, Phys. Rev. B 87, 081307 (2013).

[61] A. Molina-Sánchez and L. Wirtz, Phys. Rev. B 84, 155413 (2011).

[62] H. Li, Q. Zhang, C. C. R. Yap, B. K. Tay, T. H. T. Edwin, A. Olivier, and D. Baillargeat, Adv. Funct. Mater. 22, 1385 (2012).
[63] H. Li, Z. Yin, Q. He, H. Li, X. Huang, G. Lu, D. W. H. Fam, A. I. Y. Tok, Q. Zhang, and H. Zhang, Small 8, 63 (2012).

[64] M. Ye, D. Winslow, D. Zhang, R. Pandey, and Y. K. Yap, Photonics 2, 288 (2015).

[65] K. F. Mak, K. He, J. Shan, and T. F. Heinz, Nat. Nanotechnol. 7, 494 (2012).

[66] S. Kumar, N. McEvoy, H. Y. Kim, K. Lee, N. Peltekis, E. Rezvani, H. Nolan, A. Weidlich, R. Daly, and G. S. Duesberg, Physica Status Solidi B 248, 2604 (2011).

[67] T. Hallam, N. C. Berner, C. Yim, and G. S. Duesberg, Adv. Mater. Interfaces 1, 1400115 (2014).

[68] See Supplemental Material at http://link.aps.org/supplemental/ 10.1103/PhysRevB.98.134109 for an image of the substrate and holes.

[69] M. O’Brien, N. McEvoy, T. Hallam, H. Y. Kim, N. C. Berner, D. Hanlon, K. Lee, J. N. Coleman, and G. S. Duesberg, Sci. Rep. 4, 7374 (2014).

[70] M. Delhaye and P. Dhamelincourt, J. Raman Spectrosc. 3, 33 (1975).

[71] M. Wojdyr, J. Appl. Crystallogr. 43, 1126 (2010).

[72] S. Berciaud, S. Ryu, L. Brus, and T. Heinz, Nano Lett. 9, 346 (2009).

[73] See Supplemental Material at http://link.aps.org/supplemental/ 10.1103/PhysRevB.98.134109 for a more detailed discussion of SRIM simulations and ion interaction.

[74] J. Klein, A. Kuc, A. Nolinder, M. Altzschner, J. Wierzbowski, F. Sigger, F. Kreupl, J. J. Finley, U. Wurstbauer, A. W. Holleitner, and M. Kaniber, 2D Mater. 5, 011007 (2018).

[75] A. Castellanos-Gomez, M. Barkelid, A. M. Goossens, V. E. Calado, H. S. J. Van Der Zant, and G. A. Steele, Nano Lett. 12, 3187 (2012).

[76] A. McCreary, R. Ghosh, M. Amani, J. Wang, K. A. N. Duerloo, A. Sharma, K. Jarvis, E. J. Reed, A. M. Dongare, S. K. Banerjee, M. Terrones, R. R. Namburu, and M. Dubey, ACS Nano 10, 3186 (2016).

[77] X. Zhang, W. P. Han, J. B. Wu, S. Milana, Y. Lu, Q. Q. Li, A. C. Ferrari, and P. H. Tan, Phys. Rev. B 87, 115413 (2013).

[78] M. O'Brien, N. Scheuschner, J. Maultzsch, G. S. Duesberg, and N. McEvoy, Phys. Status Solidi B 254, 1700218 (2017).

[79] F. H. M. Rahman, S. McVey, L. Farkas, J. A. Notte, S. Tan, and R. H. Livengood, Scanning 34, 129 (2012).

[80] See Supplemental Material at http://link.aps.org/supplemental/ 10.1103/PhysRevB.98.134109 for a plot of the output values for a range of $C_{A}$.

[81] See Supplemental Material at http://link.aps.org/supplemental/ 10.1103/PhysRevB.98.134109 for a thorough description of the fitting process.

[82] A. Merrill, C. D. Cress, J. E. Rossi, N. D. Cox, and B. J. Landi, Phys. Rev. B 92, 075404 (2015).

[83] J. C. Meyer, F. Eder, S. Kurasch, V. Skakalova, J. Kotakoski, H. J. Park, S. Roth, A. Chuvilin, S. Eyhusen, G. Benner, A. V. Krasheninnikov, and U. Kaiser, Phys. Rev. Lett. 108, 196102 (2012).

[84] A. Dimiev, D. V. Kosynkin, A. Sinitskii, A. Slesarev, Z. Sun, and J. M. Tour, Science 331, 1168 (2011).

[85] T. C. Nguyen, M. Otani, and S. Okada, Phys. Rev. Lett. 106, 106801 (2011).

[86] C. T. Pan, J. A. Hinks, Q. M. Ramasse, G. Greaves, U. Bangert, S. E. Donnelly, and S. J. Haigh, Sci. Rep. 4, 6334 (2014). 
[87] See Supplemental Material at http://link.aps.org/supplemental/ 10.1103/PhysRevB.98.134109 for more details on the uncertainty calculation.

[88] J. Buchheim, R. M. Wyss, I. Shorubalko, and H. G. Park, Nanoscale 8, 8345 (2016).

[89] See Supplemental Material at http://link.aps.org/supplemental/ 10.1103/PhysRevB.98.134109 for a graph of $r_{S}$ against the maximum of the $I_{D} / I_{G}$ ratio.
[90] G. Gawlik, P. Ciepielewski, J. Jagielski, and J. Baranowski, Nucl. Instrum. Methods Phys. Res., Sect. B 406, 683 (2017).

[91] K. Yoon, A. Rahnamoun, J. L. Swett, V. Iberi, D. A. Cullen, I. V. Vlassiouk, A. Belianinov, S. Jesse, X. Sang, O. S. Ovchinnikova, A. J. Rondinone, R. R. Unocic, and A. C. T. Van Duin, ACS Nano 10, 8376 (2016). 